\title{
Patrimonio y coeducación en el marco curricular de la educación primaria
}

\author{
Laura LUCAS PALACIOS \\ Emilio José DELGADO-ALGARRA
}

Datos de contacto:

Laura Lucas-Palacios

Universidad Complutense de

Madrid

Llucas03@ucm.es

Emilio José Delgado-Algarra Centro de investigación COIDESO, Universidad de Huelva Emilio.delgado@ddcc.uhu.es

Recibido: 05/02/2021 Aceptado: 21/04/2021

\section{RESUMEN}

Los currículos educativos juegan un papel esencial en las mejoras y la innovación de la educación formal. Está en juego el acto esencial de elegir y articular lo que debe ser transmitido a cada nueva generación en nuestra relación con el pasado, pero también con el futuro que queremos construir como sociedad -democrática e igualitaria-. En esta relación el patrimonio tiene un papel significativo en la medida en que es vínculo entre pasado y futuro, así como generador de sentimiento de identidad personal y pertenencia social y cultural. Nuestra investigación tiene como objetivo analizar la forma en que se aborda el patrimonio y la coeducación en los 17 Decretos que regulan Educación Primaria en el territorio español. Los resultados muestran que, a pesar del avance que la educación patrimonial ha experimentado en esta última ley educativa como contenido en sí mismo, metodológicamente no se contempla el análisis crítico del pasado para comprender el complejo mundo en el que vivimos e incorporar la categoría de género como categoría educativa. Por otro lado, se concluye que existe regresión en materia de género en las leyes educativas derivadas de la Ley Orgánica para la Mejora de la Calidad Educativa -LOMCE-: un tema a tener en cuenta en los procesos de revisión curricular y en el diseño de programas académicos dirigidos a futuros docentes con el fin de asegurar la enseñanza de una ciudadanía igualitaria a partir de la educación patrimonial.

PALABRAS CLAVE: coeducación; enseñanza primaria; investigación cualitativa; investigación sobre el currículum; legislación educativa; patrimonio cultural. 


\title{
Heritage and coeducation in the curriculum framework of Primary Education
}

\begin{abstract}
Educational curricula play an essential role in the improvement and innovation of formal education. The act of choosing and articulating what should be transmitted to each new generation in our relationship with the past is essential, but also the future with which we want to build our society - democratic and egalitarian-. In relation to this, heritage plays a significant role as a link between past and future, as well as a generator of feeling of personal identity and social and cultural belonging. The present research aims to analyze the way in which heritage and co-education are addressed in the 17 Decrees that regulate Primary Education in the Spanish territory. The results show that, despite the progress that heritage education has experienced in this latest educational law as a content; regarding methodology, the critical analysis of the past to understand the world and to incorporate the category of gender as an educational category is not considered. On the other hand, it is concluded that there is regression in terms of gender in the educational laws derived from the Organic Law for the Improvement of Educational Quality -LOMCE-; an issue to take into account in the curricular review processes and in the design of academic programs aimed at future teachers in order to ensure education for equal citizenship based on heritage education.
\end{abstract}

KEYWORDS: co-education; primary education; qualitative research; research on the curriculum; educational laws; cultural heritage. 


\section{Introducción}

La coeducación y la educación patrimonial son contenidos didácticos que, por su carácter sociocultural, necesitan de una actualización permanente para poder adaptarse a la realidad educativa. Según García Luque y Peinado (2015), los decretos autonómicos vigentes derivados de ley educativa, Ley Orgánica 8/2013, de 9 de diciembre, para la Mejora de la Calidad Educativa (LOMCE), han mantenido y, en algunos casos, enriquecido aspectos de la ley orgánica anterior, Ley Orgánica 2/2006, de 3 de mayo, de Educación (LOE), como los relativos a la formación del profesorado en materia de género o velar por el uso de materiales basados en los valores de igualdad. Sin embargo, estos decretos también han supuesto un retroceso en cuestiones de género "haciendo desaparecer de los contenidos sociales todos aquellos relacionados con el género (diversidad afectivo-sexual, sistema sexo-género, etc.)" (García Luque y Peinado, 2015, p. 66) y afectando, por tanto, a valores fundamentales en la formación de una ciudadanía crítica e igualitaria.

Pese a la aprobación de la Ley Orgánica 3/2020, de 29 de diciembre, conocida como LOMLOE, a día de hoy, la base legislativa de los decretos y órdenes de educación primaria que se encuentran vigentes en las autonomías derivan de la ley anterior la LOMCE. En base a esta ley general, cada comunidad autónoma, conforme sus particularidades culturales, territoriales e ideológicas, establecen sus respectivas concreciones. La presente investigación tiene por objetivo principal el conocer las posibilidades didácticas que otorga cada una de las autonomías al patrimonio como contenido coeducativo; por ello, basamos nuestro análisis en la LOMCE y los 17 decretos autonómicos derivados de esta ley estatal. En un primer momento, desde la óptica de una metodología mixta, la investigación parte de un el análisis exploratorio en relación con la presencia del patrimonio y coeducación en los textos legislativos. En la segunda parte del estudio, se lleva a cabo un análisis cualitativo sobre el uso que se hace de ambos conceptos y la posible relación que se establece entre ellos.

\section{Hacia una coeducación patrimonial}

La educación patrimonial nace como un área emergente de la educación cuya complejidad radica en el hecho de que no sólo se refiere al conocimiento de elementos patrimoniales y de hechos históricos, sino también al conocimiento y construcción de la identidad individual y colectiva de la ciudadanía, además de su desarrollo como sociedad. Por lo tanto, el diseño y planificación de acciones educativas en este campo debería de centrarse en las personas (Fontal, 2016). En este sentido, la educación patrimonial puede definirse como:

“...un proceso permanente y sistemático de trabajo educacional centrado en el patrimonio cultural como fuente primaria de conocimiento y enriquecimiento individual y colectivo... La educación patrimonial es un instrumento de "alfabetización cultural" que posibilita al individuo hacer una lectura del mundo que le rodea, elevando la comprensión del universo sociocultural y la trayectoria histórico-temporal en la que está insertado. ... El conocimiento crítico y la apropiación consciente de las comunidades de su patrimonio son factores indispensables en el proceso de preservación sustentable de sus bienes, 
así como el fortalecimiento de los sentimientos de identidad y ciudadanía" (Horta, Grunberg y Monteiro, 1999, p. 6)

Es por ello que, desde nuestra perspectiva, la didáctica del patrimonio no constituye un fin en sí mismo sino que debe integrar en el proceso educativo por su relevancia en desarrollo integral de los individuos (Estepa, 2013; Lucas, 2019; Trabajo, 2017). Sin embargo, si planteamos esta visión contemporánea en los procesos educativos, debemos afrontar nuevos desafíos entre los que se encuentra la perspectiva de género.

La realidad es que el patrimonio conservado en museos y lugares históricoartísticos y arqueológicos así como el transmitido en las instituciones educativas muestra un relato histórico en el que el género masculino es el principal protagonista (Fernández Valencia, 2010; Jiménez-Esquinas, 2016; Lucas y DelgadoAlgarra, 2019). Por lo que los elementos que definen nuestra identidad cultural excluyen a la mitad de la ciudadanía o definen una ciudadanía de mujer de segunda fila, lo que Simone de Beauvoir (2005) definió como «segundo sexo». La consecuencia de esta mirada masculina es que ofrece una visión irreal y estereotipada de las mujeres aislándolas como sujetos productores de cultura y ubicándolas en espacios domésticos atemporales. Es por ello que, estamos de acuerdo con Smith en afirmar que "la herencia tiene un género" (2008, p.161); es decir, bajo una aparente neutralidad se ha naturalizado una única visión de la historia y de los artefactos vinculados a ella. La ausencia de mujeres o de lugares de importancia para la historia y las experiencias de las mujeres en el patrimonio cultural, junto al hecho de que las prácticas y valores masculinos siguen primando en nuestras sociedades patriarcales, contribuye a perpetuar las desigualdades de género en el imaginario colectivo. En este sentido, Moghadam y Bagheritari (2007) señalan que uno de los grandes obstáculos se encuentra en la búsqueda de la neutralidad cuando esta no es posible. Según estas autoras, la historia tradicionalmente conocida y los bienes patrimoniales parejos a ella otorgan más valor a las experiencias masculinas. Esto ha llevado a atribuir y difundir identidades femeninas parciales, falsas o desagradables para las mujeres. Una realidad no solo perjudicial para el género femenino, pues ellas cuentan con menos referentes con los que identificarse, experiencias de socialización distintas, discrepancias en cuanto a expectativas, diferentes valores y roles sociales, etc.; sino también para el género masculino quien construye su identidad bajo una visión limitada y estereotipada de la masculinidad, vinculada a las ideas de éxito y heroísmo (Harrison, 2012). Por su parte, Colella (2018) subraya que el criterio de la aparente objetividad científica a menudo lleva a la ceguera de género, siendo necesario diseñar un marco metodológico de género específico transversal a todas las disciplinas del currículo, que visibilice los mensajes ocultos del imaginario cultural que subyace en la sociedad española, reticente a los cambios y fruto de varios años de dictadura, represión y patriarcado. Por todo ello, a nuestro modo de ver, la educación puede y debe ser un correctivo de esta única visión de la historia y de nuestra memoria cultural. Son muchos los autores que defienden que el acto de educar significa cuestionar (Domínguez Almansa y López Facal, 2016; Kerr y Huddleston, 2016; Lucas y Delgado-Algarra, 2018), por lo que debemos apostar por una educación patrimonial que recupere al sujeto femenino como sujetos específicos de la historia y no sujetos en la historia.

En este sentido, la educación patrimonial sería clave, pues como afirma 
Fernández Valencia (2010), si incluimos la perspectiva de género en la educación patrimonial, el alumnado podrá detectar la posición social de hombres y mujeres jerarquizaciones y asimetrías- y pensar desde los de ayer para que los de hoy puedan cuestionar esas jerarquías y realizar los cambios necesarios para avanzar en igualdad. Consecuentemente, la educación patrimonial es una excelente herramienta educativa para que la ciudadanía del futuro pueda reclamar una reinvención del museo y de los lugares patrimoniales, revisar el discurso expositivo, plantearse el porqué de las obras que están expuestas y de las que no, revisar las políticas de adquisición de nuevos fondos desde la óptica de género o releer la historia. Por todo ello, y dada la importancia que tienen los currículos educativos a la hora de poner en valor los contenidos necesarios que deben adquirir nuestros estudiantes, se plantean los siguientes objetivos:

- Conocer desde una perspectiva cuantitativa la presencia o no de aspectos metodológicos y conceptuales relacionados con la coeducación y el patrimonio.

- Determinar en los distintos currículos relaciones existentes entre la educación patrimonial y la coeducación.

- Definir variables que expliquen la presencia o ausencia de estas variables y la eficiencia o no de las mismas.

\section{Método y muestra}

En la presente investigación, se opta por un enfoque metodológico mixto, partiendo de una fase cuantitativa de análisis exploratorio, seguido de una fase cualitativa. Por otro lado, se establecen como finalidades fundamentales conocer el empleo explícito que los textos curriculares autonómicos hacen de los términos patrimonio y coeducación, así como de las palabras que están asociadas a su familia léxico-semántica como patrimonial, patrimoniable, patrimonialización, coeducar, identidad, género, etc. Posteriormente, se da paso a la interpretación de la información cualitativa a través del análisis de cada caso concreto. En líneas generales, se lleva a cabo una investigación fundamentada en la metodología de análisis y comparación de contenidos (Piñuel, 2002) que, en nuestro caso, son contenidos manifiestos. La muestra que conforma nuestra investigación está formada por los 17 decretos por los que se establece el currículo de la educación primaria las respectivas autonomías y el Real Decreto 126/2014 del que derivan. La elección de la etapa de primaria se debe a que aporta una mirada crítica al futuro profesorado de primaria de las facultades de educación, pues como afirma Maldonado (2004), son el primer escalón para formar una ciudadanía igualitaria.

\section{Instrumentos de análisis y confiabilidad de los datos}

Tras un análisis exploratorio basado en la búsqueda terminológica con apoyo del programa Atlas.ti y donde la raíz utilizada fue patrim*, coeduc*, identi*, género; se analizan cualitativamente los usos que se hacen del concepto patrimonio y coeducación. En líneas generales, en relación con la fase cuantitativa, se sigue el procedimiento de trabajos similares como los de González (2011), Ferreras, Estepa y Wamba (2010), Oriola (2017) y Pinto y Molina (2015); mientras en la fase cualitativa se toman como referentes trabajos como los de Arias (2017) o Lucas y 
Delgado-Algarra $(2018,2019)$. Los documentos curriculares fueron analizados por dos expertos en estudios de género y educación patrimonial, integrantes del grupo de investigación DESYM de Huelva y agentes activos en el proyecto de investigación EPITEC -Educación Patrimonial para la inteligencia territorial y emocional de la ciudadanía- (MINECO/FEDER, con código de referencia EDU2015-67953-P). Por su parte, los datos se agruparon como se presenta en la tabla 1.

\section{Tabla 1.}

Variables cualitativas del análisis comparativo de los documentos legislativos

\begin{tabular}{lll}
\hline ANÁLISIS & Relación entre patrimonio & Variable destinada a conocer qué \\
EXPLORATORIO: & e identidad & $\begin{array}{l}\text { relación establecen los currículos de } \\
\text { VARIABLES }\end{array}$ \\
CUALITATIVAS & Relación entre patrimonio & Variable para detectar si existen \\
& y coeducación & $\begin{array}{l}\text { referencias concretas en relación con } \\
\text { la coeducación y se relacionan de }\end{array}$ \\
& manera explícita o no con la \\
& educación patrimonial.
\end{tabular}

Fuente: elaboración propia.

\section{Resultados y discusión}

Los resultados cuantitativos a partir de la búsqueda terminológica quedan recogidos en la Tabla 2. En la primera fila se muestra el Real Decreto por el que se establece el currículo básico de la educación primaria, seguido de todos los decretos autonómicos. Y en las columnas se recoge el número de veces que se emplea el término patrimonio (y derivados), coeduca* (y derivados), identidad y género.

\section{Tabla 2}

Resultados de la búsqueda terminológica en los documentos legislativos

\begin{tabular}{|c|c|c|c|c|}
\hline \multicolumn{5}{|c|}{ CURRÍCULUM OFICIAL [ÁMBITO NACIONAL] } \\
\hline & Patrim* & Coeduca* & Identidad & Género \\
\hline $\mathrm{RD}$ & 16 & 0 & 6 & 14 \\
\hline \multicolumn{5}{|c|}{ CURRÍCULUM OFICIAL [ÁMBITO AUTONÓMICO] } \\
\hline & Patrim* & Coeduca* & Identidad & Género \\
\hline AND & 206 & 5 & 163 & 125 \\
\hline ARG & 58 & 0 & 4 & 41 \\
\hline AST & 136 & 1 & 38 & 68 \\
\hline BAL & 36 & 3 & 19 & 40 \\
\hline CAN & 79 & 9 & 74 & 97 \\
\hline CANT & 53 & 0 & 20 & 39 \\
\hline CLM & 69 & 0 & 24 & 65 \\
\hline
\end{tabular}

70 ISSN0213-8646|E-ISSN2530-3791• RevistaInteruniversitariadeFormación delProfesorado,96 (35.2) (2021), 65-82 


\begin{tabular}{lllll}
\hline CYL & 72 & 0 & 24 & 41 \\
CAT & 19 & 0 & 9 & 26 \\
EXT & 78 & 0 & 26 & 32 \\
GAL & 30 & 0 & 15 & 51 \\
LRIO & 13 & 0 & 14 & 65 \\
MAD & 11 & 0 & 11 & 24 \\
MUR & 35 & 0 & 15 & 41 \\
NAV & 16 & 0 & 14 & 93 \\
PV & 15 & 9 & 55 & 27 \\
VAL & 112 & 4 & 28 & 121 \\
TOTAL & 1054 & 31 & 559 & 1010 \\
\hline
\end{tabular}

Note RD = Real Decreto; $\mathrm{ARG}=$ Aragón; $\mathrm{AST}=$ Asturias; $\mathrm{BAL}=$ Baleares; $\mathrm{CAN}=$ Canarias; CANT = Cantabria, CLM = Catilla la Mancha; CYL = Castilla y León; Cataluña; Ext = Extremadura; GAL = Galicia; LRIO = La Rioja; MAD = Madrid; MUR = Murcia; NAV = Navarra; PV = País Vasco; VAL = Valencia. Fuente: elaboración propia.

En primer lugar, el término que más se repite es patrimonio, siendo el currículo andaluz el que más veces itera este término. También es esta norma educativa la que menciona con más asiduidad la palabra identidad. Ahora bien, se hace necesario realizar una exploración por las diferentes áreas de currículo para que un análisis más riguroso (figura 1).

\section{Figura 1}

Uso del concepto patrimonio en el Decreto autonómico de Andalucía para educación primaria [Atlas.Ti]

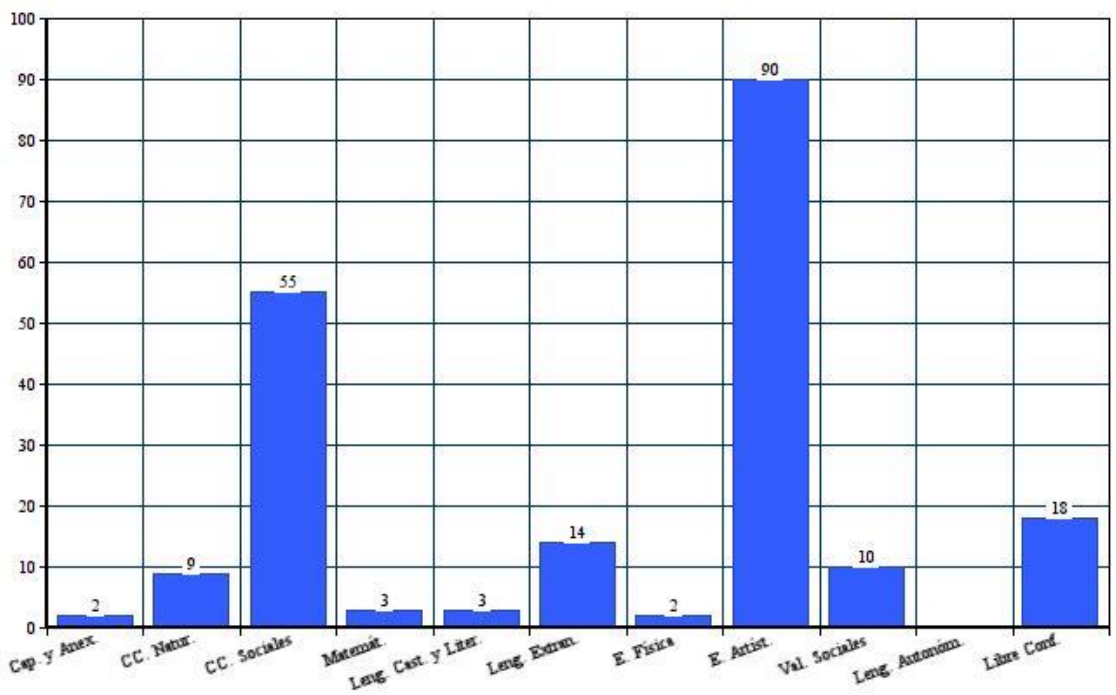

Fuente: elaboración propia 
Mientras que el área de Ciencias de la Naturaleza (donde la palabra patrimonio aparece 9 veces) hace referencia a desarrollar la capacidad de valorar, respetar y conservar el medio natural, lo más interesante de esta búsqueda es que, en el área Educación Artística (90 veces) y en el área de Ciencias Sociales (55 veces), patrimonio e identidad están íntimamente relacionados. Sin embargo, lejos de considerar el patrimonio de manera explícita como un elemento identitario para reconocer los estereotipos y rechazar las situaciones de injusticia y de discriminación por motivos de género, orientación afectivo-sexual o cualquier otro motivo que lleve a una sociedad no igualitaria, el concepto de identidad que define se basa en lo que Lucas (2018) ha llamado "ciudadano personalmente responsable" es decir, aquel que actúa de manera responsable en su comunidad, recogiendo basura, donando sangre, reciclando, ofreciéndose como voluntario y manteniéndose libre de deudas; pero que carece del pensamiento crítico de reconocer las desigualdades e injusticias estructurales de la comunidad a la que pertenece.

Por otro lado, el currículo andaluz también es la norma que más veces utiliza el término género; sin embargo, se refiere a una categoría de algún aspecto concreto como pueden ser los géneros literarios o musicales. Sólo alude explícitamente a la violencia de género en los objetivos del área de lengua y literatura, en relación con detectar este tipo de violencia en los anuncios publicitarios (Decreto 97/2015, 3 de marzo, p.133); y en el área de Educación Física en referencia al juego limpio, “evitando discriminaciones por razones de género" (Decreto 97/2015, 3 de marzo, p. 489).

Por lo que respecta al resto de comunidades, el término de patrimonio aparece mayormente en el área de Ciencias Sociales y de Educación Artística. También el de identidad, pero haciendo referencia al conocimiento y reconocimiento de los elementos patrimoniales como hechos diferenciadores de su comunidad autónoma para la construcción de una identidad social. En general, en todas las normas autonómicas, el patrimonio parece tener como principal finalidad educativa formar en valores pasivos (respetar, valorar, reconocer la importancia...), pero no se considera como una herramienta educativa de análisis histórico y social para comprender las desigualdades del presente y desarrollar actitudes que lleven a erradicar estas diferencias. Además, a pesar de que el concepto de patrimonio que proyectan la mayoría de los textos autonómicos es bastante amplio, incluyendo el patrimonio etnológico, natural, científico o tecnológico, histórico y artístico, no se explicita -ni siquiera en las orientaciones y ejemplificaciones de cada etapa- ninguna actividad que muestre la invisibilización de las actividades de las mujeres a lo largo de la historia. Una invisibilización que dejaría de serlo con una didáctica del patrimonio inmaterial en el que el alumnado pudiera analizar la importancia de los cuidados y de las actividades que históricamente han recaído en el género femenino para la evolución de la sociedad. Ocupaciones que, como afirman González Marcén y Picazo (2005), constituyen el tejido temporal y de relación del ciclo de las estructuras cotidianas que articulan y transforman las prácticas de convivencia y su devenir histórico. Un hecho este que, en el caso del currículo murciano, es más llamativo pues no aparece ni una sola mención al patrimonio inmaterial.

En el lado opuesto encontramos el decreto canario, siendo el único documento normativo que utiliza el concepto de educación patrimonial para referirse a su valor didáctico como fuente para el conocimiento del pasado y para desarrollar una visión crítica del mundo y de sus transformaciones en el tiempo y en el espacio. Así, 
podemos deducir que la finalidad educativa que esta norma otorga al patrimonio es que el alumnado pueda experimentar nuevas formas de sentir, pensar y actuar en una sociedad global y contribuir al desarrollo de un mundo más justo, solidario y sostenible. Destaca la importancia que da al patrimonio como elemento de reflexión y, sobre todo, recoge de manera explícita la contribución de las mujeres al patrimonio canario. Concretamente, queda recogido en el bloque de aprendizaje IV: Las huellas del tiempo, en los criterios de evaluación:

“También será objeto de evaluación que el alumnado reconozca algunas obras de nuestro patrimonio artístico, cultural e histórico e identifique y valore la contribución social de los hombres y las mujeres que han realizado estas producciones (artísticas, culturales), redactando trabajos (pequeñas monografías, biografías, presentaciones...) a partir del tratamiento de distintas fuentes y formatos (orales, películas, imágenes, reportajes, entrevistas, internet...), exponiendo sus resultados y el proceso seguido" (D 89/2014, 1 de agosto, p. 22033)

La otra disciplina en la que encontramos la palabra patrimonio repetida más veces es, como hemos dicho anteriormente, la Educación Artística. Y dentro de esta materia, las distintas autonomías -en mayor o menor medida- incluye con más o menos relevancia el concepto de patrimonio en los bloques de contenidos: "Expresión Artística”, "Escucha” y "La Música, el Movimiento y la Danza". En estos bloques se otorga un peso especial al conocimiento y el respeto del patrimonio cultural como nexo indiscutible entre las diferentes artes y culturas, la educación integral y la identidad personal y social. Además, se insiste en la idea de respetar y conservar. Algunos decretos como el catalán y de nuevo el canario, dan un paso más, poniendo el acento en fomentar la participación activa del alumnado mediante la experimentación auditiva y/vocal para que los/as estudiantes puedan desarrollar un pensamiento crítico para poder analizar el arte contemporáneo actual. Sin embargo, a pesar de esta buena declaración de intenciones reflejada en la introducción y anexos del área, no se plasman en acciones concretas en cuanto al uso del patrimonio como herramienta educativa para poner en evidencia la discriminación que las mujeres sufren actualmente en canciones, películas y todas las artes contemporáneas en general.

Si seguimos avanzando en nuestro análisis, cabe destacar que, a pesar de que la gran mayoría de las administraciones utilizan el patrimonio principalmente en estas dos asignaturas que acabamos de analizar, existen tres autonomías en las que el patrimonio ocupa un lugar relevante en otras materias: Asturias, Valencia y País Vasco. En el caso de Asturias, son las asignaturas de Lengua Asturiana y Literatura y la asignatura de libre configuración Cultura Asturiana. Tanto en una materia como en otra, el patrimonio es utilizado como una herramienta educativa para "asimilar", "integrar", "respetar" y "valora" la cultura de la que forman parten. Y a pesar de que en el texto introductorio, sí que hace mención a la oportunidad de "reflexionar sobre los valores que ayudan a la formación de la personalidad y a la integración social, partiendo de la convicción de que la cultura humana siempre es una realidad activa en el proceso de socialización" (D 82/2014, 28 de agosto, p.387), nuevamente, esta interesante idea de entender el proceso socializador de manera dinámica y otorgando a las personas la capacidad de agencia para transformar y construir una sociedad más igualitaria y justa, se queda sin materializar a nivel práctico. De acuerdo con la teoría de la socialización diferencial de género (Ferrer et. alt. 2006; 
Simon, 2010), niños y niñas en su proceso de iniciación a la vida social y cultural a partir de los agentes de socialización (familia, escuela, medios de comunicación, etc.), adquieren una identidad diferenciada que conlleva entre otros aspectos a asimilar normas, actitudes y códigos diferenciados según el sexo. Según Subirats (2014), los mensajes que transmiten los contenidos manifiestos del currículo, pero también los contenidos del currículo oculto, son interiorizados por los niños y las niñas, adquiriendo una identidad diferenciada que conlleva entre otros aspectos a asimilar normas, actitudes y códigos distintos según el sexo. La investigación de Ortega-Sánchez (2019) con profesorado de educación primaria destaca el potencial que la mejora de la visibilidad de las mujeres en el discurso histórico - social tiene en la educación para la ciudadanía democrática; destacando la importancia de que los planes de estudio incluyan la capacidad crítica frente a ideologías y discursos vinculados con concepciones androcéntricas de la realidad. En este sentido, a nuestro modo de ver, además de visibilizar y cuestionar estos condicionamientos es necesario que desde las escuelas se ofrezca la posibilidad de plantear nuevos modelos de feminidad y masculinidad que rompan las estructuras tradicionales de la cultura patriarcal de la que formamos parte; siendo la educación patrimonial un buen recurso para que el alumnado pueda investigar, analizar y cuestionar los mandatos hegemónicos.

Por su parte, el decreto valenciano también tiene una asignatura de libre configuración sobre la cultura autonómica. En este caso el patrimonio se relaciona con el concepto de identidad social, priorizando de nuevo la enseñanza de una cultura lineal y progresiva, caracterizada según Levy, "por el privilegio blanco, masculino, elitista, heterosexual, imperialista y colonial” (2013, p.90). En cuanto a la normativa del País Vasco, llama la atención que el empleo de la palabra patrimonio se concentre en la parte de introducción y en los anexos y no se integre como contenido de las asignaturas, tal y como ocurre en el resto de autonomías. Esto es porque esta norma, estructura el aprendizaje en torno a las competencias clave y son las asignaturas las que se plantean de manera trasversal. En consecuencia, nos encontramos ante una realidad contradictoria, ya que mientras la Ley Orgánica $3 / 2007$, de 22 de marzo, para la igualdad efectiva de mujeres y hombres, aboga por una educación para la igualdad de género, la materialización de acciones para su consecución sigue siendo escasa y con un limitado acierto en su gestión. En líneas generales, en convergencia con los resultados de investigación de Martínez Rodríguez y Fontal (2020) existen diferencias significativas en cuanto a la conciencia patrimonial y la cobertura curricular.

Respecto al término coeducación es llamativo que solo se repita 9 veces en las dos comunidades que más lo mencionan: Canarias y País Vasco. En el decreto canario, la utilización de este término se concentra en la materia de Lengua y Literatura. Concretamente, se hace referencia a él en 8 ocasiones dentro del bloque "El alumnado como agente social" y una única vez en los objetivos generales:

La eliminación de los prejuicios, estereotipos y roles en función del sexo o de la orientación sexual, la integración del saber de las mujeres y su contribución social e histórica al desarrollo de la humanidad, la prevención de la violencia de género y el fomento de la coeducación (D 89/2014, 1 de agosto, p.22062)

En cuanto a la legislación vasca, como ya hemos explicado en líneas anteriores, se organiza el aprendizaje en torno a las competencias clave. Así que de nuevo el concepto coeducación se centraliza en la primera parte del currículo, es decir en la 
introducción; y sólo una vez se utiliza en la parte de las competencias, «competencia para vivir», y dentro de esta, en el ámbito de la Educación Física.

Solo comunidad de Andalucía relaciona coeducación y patrimonio, aunque sea de manera indirecta. Lo hace en la asignatura de Ciudadanía y Derechos Humanos. En uno de sus criterios de evaluación, promueve los debates acerca del papel del hombre y la mujer en la sociedad actual y la investigación sobre la evolución de dicho papel. Aunque no lo manifieste de manera explícita, investigaciones recientes (Lucas y Delgado-Algarra, 2018; Segura, 1997; Seva, Soriano y Vera, 2010; Tomé, 2017) demuestran que una de las maneras de investigar sobre los roles de género a lo largo de la historia es a través del patrimonio.

Por último, debemos de mencionar que la norma estatal de la que deriva todas las leyes educativas, la LOMCE, no menciona ni una sola vez el término coeducación (figura 2). Además, en la LOMCE no se hace ninguna mención a respetar y reconocer las diversidades de género y afectivo-sexuales existentes en nuestra sociedad, como sí lo hiciera la antigua LOE 2/2006. De este modo, contenidos que formaban parte del currículo manifiesto de la ley anterior pasan ahora, en el mejor de los casos, a ser parte del currículo oculto. Un hecho que reafirma la idea que enuncian Planella y Pié acerca de las técnicas de exclusión en pedagogía: "se excluyen individuos, identidades, prácticas, así como formas de construir el conocimiento” (2012, p.275).

\section{Figura 2}

Nube de palabras generada a partir del Real Decreto 126/2014, por el que se establece el currículo básico de la educación primaria, LOMCE, obtenida mediante el software Atlas.Ti.

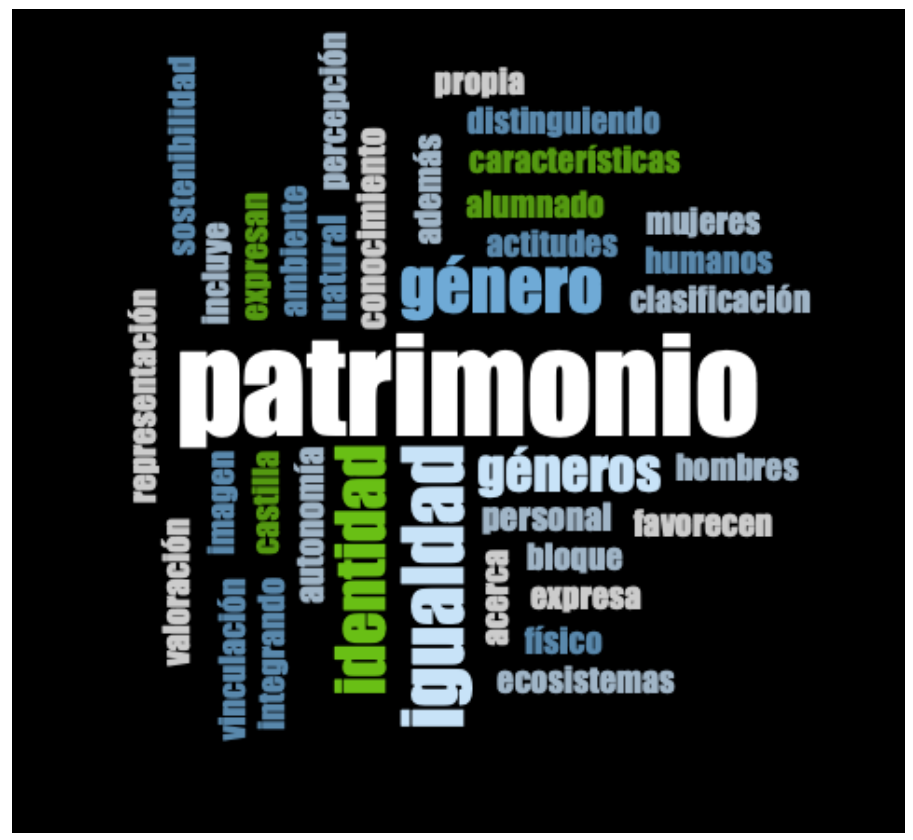

Fuente: elaboración propia 
A pesar de la amplía tipología de patrimonio que se incluye dentro de los currículos, incluyendo además del histórico-artístico, el patrimonio natural, lingüístico, arqueológico, científico, tecnológico, etc. la ausencia de las experiencias de las mujeres en el patrimonio es relevante. Son muchas las investigaciones (Adams, 2010; Arvin y Arvin, 2010; Chidgey, 2012; De la Peña, 2007) que han manifestado la necesidad de reflexionar desde un pensamiento crítico sobre la causalidad del colectivo femenino en el imaginario y en cómo se ha transmitido y sigue trasmitiendo la ideología patriarcal a través del patrimonio. Este es un aspecto clave para asegurar un enfoque sistémico de la educación del patrimonio (Cuenca et alt., 2017), donde los diferentes tipos de patrimonio deben entenderse como las diferentes partes de un mismo concepto. Por lo que consideramos necesario que los valores coeducativos que todas las autonomías manifiestan, en forma de buenos propósitos en sus introducciones, se materialicen de forma efectiva y explícita. En este sentido y de nuevo en sintonía con otras investigaciones publicadas (Fontal y Gómez-Redondo, 2016; Martínez y Fontal, 2020; Pinto y Molina, 2015) observamos que en la legislación educativa española hay un predominio de visiones estéticas y temporales que involucran metodologías tradicionales y académicas, así como con fines de conservación (Cuenca et al., 2017). Como indica Coperland (2006), al enfoque debe evolucionar hacia un enfoque globalizador y simbiótico que fomente procesos relacionales entre la propiedad cultural y el estudiante para construir significados propios. Por otro lado, en contraste algunos de los resultados alcanzados tras el análisis de la normativa estatal y de los currícula autonómicos, en el escenario internacional, la posición que las convenciones de la UNESCO (1972, 2003, 2014) y el Consejo de Europa que llevan varias décadas defendiendo incluye que

El patrimonio cultural en su conjunto constituye una fuente compartida de recuerdo, comprensión, identidad, cohesión y creatividad, y de ideales, principios y valores, derivados de la experiencia adquirida a través del progreso y los conflictos pasados, que favorecen el desarrollo de una situación pacífica y estable sociedad (Consejo de Europa, 2005, art. 3)

Además, sostiene que la educación patrimonial asegura procesos de empoderamiento social que nos permiten ser más competentes ante los retos de un futuro incierto. Por eso es fundamental emprender una revisión con el fin de mejorar los planes curriculares que conduzcan a la discusión sobre si el patrimonio cultural debe incluirse en el diseño educativo como un tema por derecho propio (Barghi et alt., 2017; Hang et alt., 2017; Hunter, 1988) o como fuente fértil para el estudio de otros sujetos (Fuhai, 2017). Si pretendemos educar para la igualdad de género, difícilmente podremos conseguirlo desde los discursos patriarcales, siendo la educación patrimonial una excelente herramienta didáctica para cuestionar estos discursos y garantizar la perspectiva y la equidad de género en las aulas de nuestro país.

\section{Conclusiones}

El primer objetivo del trabajo era indagar sobre los aspectos cuantitativos de los currículos de Primaria en relación con las palabras patrimonio, coeducación e identidad. Se pueden concluir dos grandes rasgos. En primer lugar, observamos que en la nueva legislación el patrimonio cultural pasa de ser un recurso a ser 
considerado un contenido que las diferentes áreas incorporan de manera transversal, para conseguir desarrollar las competencias clave. Sin embargo, la mayoría de las autonomías, basándose en los criterios mínimos establecidos por el Real Decreto 126/2014, se limitan a incluirlo únicamente en las Ciencias Sociales y en la Educación Artística sin llegar a profundizar en el concepto ni establecer vínculos con su capacidad identitaria; perdiendo así, todo el potencial que la educación patrimonial tiene como contenido coeducativo. Solo Andalucía y Asturias lo integran en todas las asignaturas, superando los mínimos establecidos por el Real Decreto. Pero incluso, en el caso de Andalucía, en el que veíamos que patrimonio e identidad están íntimamente relacionados, el concepto de identidad que desarrolla el texto legislativo de esta comunidad está muy limitado, enfocándose únicamente a la adquisición de "conocer las manifestaciones más significativas que forman parte del patrimonio cultural que caracterizan España". La pregunta que nos surge es ¿sólo con «conocer» los bienes culturales se adquieren las competencias necesarias para desarrollar una identidad de pertenencia a una comunidad? Y si es así, ¿qué tipo de identidad se asimila?

La función que recibe el patrimonio por parte de los decretos autonómicos está siendo desaprovechada. Como hemos visto, la gran mayoría lo consideraban como un importante recurso didáctico para el aprendizaje de múltiples conocimientos: conceptuales, procedimentales, actitudinales; incluyéndolo en mayor o menor grado en diferentes asignaturas, pero este uso educativo que se hace de él se limita a «conocer», «estudiar», «comprender», «respetar», «conservar», «legar», etc., es decir, se utiliza el patrimonio como un recurso para la asimilación de una identidad social homogénea y sin género. Pero, a nuestro modo de ver, esta propuesta es engañosa, cualquier grupo social está formado por personas con identidades diferentes que deben ser respetadas e integradas por igual. Reconocer que hombres y mujeres (y posiblemente personas de otros géneros) participaron en cada experiencia humana que da lugar a un bien patrimonial es la clave para garantizar la equidad de género en los discursos educativos. En definitiva, la legislación vigente camufla entre sus líneas de progreso educativo y declaración de buenas intenciones, una grave involución de los derechos de las mujeres. La coeducación es tratada como un contenido trasversal pero que no se concretiza en objetivos ni contenidos didácticos, perdiéndose como categoría de análisis y lo más importante como un contenido vivencial y educativo transformador de la sociedad. El currículo siempre ha estado expuesto a tensiones políticas en la medida en que articula el orden social presente y futuro, ya que los planes educativos determinan cómo, cuándo y lo que las personas deberían aprender y dar forma al futuro de la sociedad.

\section{Referencias}

Adams, R. (2010). The New Girl in the Old Boy Network: Elizabeth Esteve-Coll at the Victoria and Albert Museum. En A. K. Levin (Ed.). Gender, Sexuality and Museum. London: Routledge, 28-42.

Arias, N. (2017). Educando para una sociedad integradora. En R. De la Fuente y C. Munilla (Eds.). Patrimonio y creatividad miradas educativas. Valladolid: Verdelis, 309-319

Arvin, H. L. y Arvin, A. H. (2010). The linear Heritage of Women. Bloomington: 
Universe.

Barghi, R.; Zakaria, Z.; Hamzah, A. y Hashim, N. (2017) Heritage education in the Primary School Standard Curriculum of Malaysia, Teacher and teaching education, 61, 124-131. https://doi.org/10.1016/j.tate.2016.10.012

Beauvoir, S. (2005). El segundo sexo. Madrid: Cátedra, Universitat de València. Chidgey, R. (2012). Feminist Memory (Word Press). From a Research Project Looking at Feminist Memory, Media and Archives Retrieved from http://feministmemory.wordpress.com/links/

Colella, S. (2018). Not a mere tangential outbreak: gender, feminism and cultural heritage. Il Capitale culturale, 18, 251-275. https://doi.org/10.13138/2039-2362/1897

Coperland, T. (2006). European democratic citizenship, heritage education and identity. Strasbourg, France: Council of Europe.

Council of Europe. (2005) Council of Europe Framework Convention on the Value of Cultural Heritage for Society (Faro, 27.X.2005). Council of Europe Treaty $\quad$ Series, $199 . \quad$ Retrieved from http://conventions.coe.int/Treat y/EN/Treat ies/Html/199.htm

Cuenca, J. M.; Estepa, J.; y Cáceres, M. (2017) Heritage, education, identity and citizenship. Teachers and textbooks in compulsory education. Revista de Educación, 375, 136-159. https://doi.org/10.4438/1988-592X-RE2016-375-338

Decreto 89/2014, 1 de agosto, por el que se establece la ordenación y el currículo de la Educación Primaria en la Comunidad Autónoma de Canarias (BOC num. 156, 13/08/2014).

Decreto 89/2014, 28 de agosto, por el que se establece la ordenación y el currículo de la Educación Primaria en el Principado de Asturias (BOPA num. 202, 30/08/2014).

Decreto $97 / 2015,3$ de marzo, por el que se establece la ordenación y el currículo de la Educación Primaria en la Comunidad Autónoma de Andalucía (BOJA núm. 50, 13/03/2015).

De la Peña, E. M. (2007). Fórmulas para la igualdad n.o 5 [en línea]. Madrid. Fundación Mujeres. Recuperado de www.fundacionmujeres.es/maletincoeducacion/pdf/CUAD5vert.pdf

Domínguez Almansa, A. y López Facal, R. (2016). Memoria histórica, patrimonio y formación del profesorado de educación primaria. En: Sebastian Molina, Naira LLonch y Tania Martínez Gil (eds.). Identidad, ciudadanía y patrimonio: educación histórica para el siglo XXI. Gijón: Trea.

Estepa, J. (2013). La educación patrimonial en la escuela y el museo: investigación y experiencias. Huelva.

Fernández Valencia, A. (2010). Género e historia: una perspectiva didáctica. En M. J. Clavo Sebastián, M. A. Goicoechea, A. Fernández Valencia, J. M. López y N. Martínez (Eds.). Las mujeres en la enseñanza de las Ciencias Sociales. Madrid: Síntesis, 61-91.

Ferrer, V.; Bosch, E.; Ramis, Mํㅡ C.; Torres, G. y Navarro, C. (2006). La violencia contra las mujeres en la pareja: creencias y actitudes en estudiantes universitarios/as. Psicothema, 18 (3), 359-366. 
Ferreras, M.; Estepa, J. y Wamba, A. Ma (2010). El patrimonio en el área de Conocimiento del Medio de Educación Primaria. Análisis curricular. En R.M. Ávila, M. P. Rivero y P. L. Domínguez (Eds.). Metodología de investigación en Didáctica de las Ciencias Sociales. Zaragoza: Universidad de Zaragoza, 293-301.

Fontal, O. (2016). El patrimonio a través de la educación artística en la etapa de primaria. Arte, Individuo y Sociedad, 28 (1), 105-120. https://doi.org/10.5209/rev ARIS.2016.v28.n1.47683

Fontal, O. y Gómez-Redondo, C. (2016) Heritage education and heritagization processes: SHEO Metodology for Educational Programs Evaluation, Interchange, 47(1), 65-90. https://doi.org/10.1007/s10780-015-9269$\mathrm{z}$

Fuhai, A. (2017) The basis for integrating local knowledge into the school curriculum for Tibetans in Southern Gansu. Chinese Education \& Society, 50(1), 12-17. https://doi.org/10.1080/10611932.2016.1262182

García Luque, A. y Peinado, M. (2015). LOMCE: ¿es posible construir una ciudadanía sin la perspectiva de género?, IBER. Didáctica de las Ciencias Sociales, 79, 65-72.

González, N. (2011). La presencia del patrimonio cultural en los currícula de educación infantil, primaria y secundaria obligatoria en España. Patrimonio cultural de España, 5, 58-75.

González Marcén, P. y Picazo Gurina, M. (2005). Arqueología de la vida cotidiana. En M. Sánchez Romero (Ed.). Arqueología y género, 141-159. Granada: Universidad de Granada.

Hang, N. V. T.; Meijer, M. R.; Bulte, A. y Pilot, A. (2017). Designing a primary science curriculum in a globalizing world: How do social constructivism and Vietnamese culture meet? Cultural Studies of Science Education, 12(3), 739-760. https://doi.org/10.1007/s11422-015-9696-2

Harrison, R. (2012). Heritage: Critical Approaches. London: Routledge.

Horta, M. L.; Grunberg, E. y Monteiro, A. (1999). Guía básica de educação patrimonial. Brasilia: Instituto do Patrimõnio Histórico e Artístico Nacional.

Hunter, K. (1988). Heritage education in the social studies. Bloomington: IN, Information Analysis.

Jiménez-Esquinas, G. (2016). De añadir mujeres y agitar a la despatriarcalización del patrimonio: la crítica patrimonial feminista, Revista PH, 89, 137-140.

Kerr, D. y Huddleston, T. (2016). La enseñanza de temas controvertidos. Madrid: Ministerio de Educación Cultura y Deporte.

Ley Orgánica 8/2013, de 9 de diciembre, para la Mejora de la Calidad Educativa. Boletín Oficial del Estado, núm. 295.

Levy, J. (2013). Gender, Feminism, and Heritage. En P. Bihel (Ed.). Heritage in the Context of Globalization: Europe and the Americas, 85-94. London: C. Prescott.

Lucas, L. (2018). La enseñanza del patrimonio y de la ciudadanía en las clases de Ciencias Sociales. Un estudio de caso en ESO. Tesis doctoral. Universidad de Huelva.

Lucas, L. (2019). Otra educación es posible: cuando el patrimonio se convierte 
en piedra filosofal de la educación. Interamericana de España: McGrawHill.

Lucas, L. y Delgado-Algarra, E. (2018). Educación para una ciudadanía comprometida en la enseñanza de las Ciencias Sociales: ¿Qué piensa el alumnado de un profesor innovador sobre su aprendizaje? Didáctica de las Ciencias Experimentales y Sociales, 35, 3-16.

Lucas, L. y Delgado-Algarra, E. (2019). La coeducación en la educación patrimonial. Resultados de un estudio de caso. Enseñanza de las Ciencias Sociales, 18, 5-15.

Maldonado, L. (2004). El alumnado de Magisterio ante la Historia de las Mujeres: percepción y actitudes. En M. I. Vera y D. Pérez i Pérez (Coords.), Formación de la ciudadanía: las TICs y los nuevos problemas. Alicante: Asociación Universitaria de Profesores de Didáctica de las Ciencias Sociales. en: https://dialnet.unirioja.es/servlet/libro?codigo=8688

Martínez Rodríguez, M. y Fontal, O. (2020). Dealing with heritage as Curricular Content in Spain's Primary Education. Curriculum Journal, 31 (1), 77-96.

Moghadam, V. y Bagheritari, M. (2007). Cultures, Conventions and the Humans Rights of Women: Examining the Convention for Safeguarding Intangible Cultural Heritage and the Declaration on Cultural Diversity. Museum International, 59, 9-18.

Oriola, S. (2019). Patrimonio y educación patrimonial en el marco legislativo de la educación primaria. Profesorado. Revista de Currículum y Formación de Profesorado, 23(3), 535-553. DOI: https://doi.org/10.30827/profesorado.v23i3.11242

Ortega-Sánchez, D. (2019). Teaching Gender in the History Classroom: An Investigation into Initial Training of Primary Education Teachers. Education Sciences, 9 (2). https://doi.org/10.3390/educsci9020114

Pinto, H. y Molina, S. (2015). La educación patrimonial en los currículos de ciencias sociales en España y Portugal. Educatio Siglo XXI, 33 (1), 103128. https://doi.org/10.6018/j/222521

Piñuel, José Luis (2002). Epistemología, metodología y técnicas del análisis de contenido. Estudios de Sociolingüística (3), 1, 1-42. DOI: https://doi.org/10.6018/i/222521

Planella, J. y Pié, A. (2012). Pedagoqueer: resistencias y subversiones educativas. Educación XXI, 15 (1), 265-283.

Segura Graino, C. (1997): La Historia de las mujeres en el nuevo paradigma de la Historia. Madrid: Al-Mudayna.

Seva, F.; Soriano, M. C. y Vera, M. I. (2010). La práctica docente en Ciencias Sociales: un análisis cualitativo. En R. M. Ávila, María P. Rivero, y P. L. Domínguez (Eds.). Metodología de investigación en didáctica de las Ciencias Sociales. Zaragoza: Institución "Fernando el Católico".

Simón, M. E. (2010). La igualdad también se aprende. Cuestión de educación. En N. Solsona, A. Tomé, R. Subías, J. Pruna y X. De Miguel (2005). Cuinar, planxar i tenir cura d'altri a l'escola. Barcelona: Octaedro.

Smith, L. (2008), Heritage, Gender and Identity. En B. Graham y P. Howard (Eds.). The Ashgate Research Companion to Heritage and Identity, 159178. Aldershot: Ashgate. 
Subirats, M. (2014). La LOMCE: hacia una educación antidemocrática. Revista Interuniversitaria de Formación del Profesorado, 81, 45-57

Tomé, A. (2017). Estrategias para elaborar proyectos coeducativos en las aulas. ATLÁNTICAS - Revista Internacional de Estudios Feministas, 2 (1), 89-116. https://doi.org/10.17979/arief.2017.2.1.1979

Trabajo, M. (2017). Educación patrimonial como vínculo entre escuela y museo: un estudio de caso en ciencias sociales de ESO. Tesis doctoral. Universidad de Huelva.

UNESCO. (1972) Convention for the protection of the world cultural and natural heritage (Paris, UNESCO). Retrieved from https://whc.unesco.org/en/conve ntion text/

UNESCO. (2003) The convention for the safeguarding of the intangible cultural heritage (Paris, UNESCO). Retrieved from https://unesdoc.unesco.org/ark:/48223/pf0000132540

UNESCO. (2014) Managing cultural world heritage (Paris, UNESCO). Retrieved from https://whc.unesco.org/en/manag ing-cultu ral-world-herit age/ 
82 ISSN0213-8646|E-ISSN2530-3791 • RevistaInteruniversitariadeFormación delProfesorado,96 (35.2) (2021), 65-82 